\title{
Optimal Lymph Node Yield for Survival Prediction in Rectal Cancer Patients After Neoadjuvant Therapy
}

\begin{abstract}
Yu-Min Lin,' Chia-Lin Chou, ${ }^{2,3}$ Yu-Hsuan Kuo, ${ }^{4,5}$ Hung-Chang $\mathrm{Wu}^{4,6}$ Chia-Jen Tsai, ${ }^{7}$ Chung-Han Ho, (1D ${ }^{8,9}$ Yi-Chen Chen, (1D ${ }^{8}$ Ching-Chieh Yang, $\mathbb{D}^{6,7, *}$ Cheng-Wei Lin ${ }^{10-12, *}$

'Division of Hepatogastroenterology, Department of Internal Medicine, Chi Mei Medical Center, Tainan, Taiwan; ${ }^{2}$ Division of Colorectal Surgery, Department of Surgery, Chi Mei Medical Center, Tainan, Taiwan; ${ }^{3}$ Department of Medical Laboratory Science and Biotechnology, Chung Hwa University of Medical Technology, Tainan, Taiwan; ${ }^{4}$ Division of Hematology and Oncology, Department of Internal Medicine, Chi Mei Medical Center,

Tainan, Taiwan; ${ }^{5}$ Department of Cosmetic

Science, Chia-Nan University of Pharmacy and Science, Tainan, Taiwan; ' ${ }^{6}$ Department of Pharmacy, Chia-Nan University of Pharmacy and Science, Tainan, Taiwan; ${ }^{7}$ Department of Radiation Oncology, Chi Mei Medical Center, Tainan, Taiwan; ${ }^{8}$ Department of Medical Research, Chi Mei Medical Center, Tainan, Taiwan; 'Department of Information Management, Southern Taiwan University of Science and Technology, Tainan, Taiwan;

${ }^{10}$ Department of Biochemistry and Molecular Cell Biology, School of Medicine, College of Medicine, Taipei Medical University, Taipei,

Taiwan; ' 'Graduate Institute of Medical Sciences, College of Medicine, Taipei Medical University, Taipei, Taiwan; ${ }^{2}$ Cell Physiology and Molecular Image Research Center, Wan Fang Hospital, Taipei Medical University, Taipei, Taiwan

*These authors contributed equally to this work
\end{abstract}

Correspondence: Ching-Chieh Yang Department of Radiation Oncology Chi-Mei Medical Center, No. 90I,

Zhonghua Road, Yung Kang District, Tainan

City, 70I, Taiwan, Republic of China

Tel +886628 I 28 I I

Email cleanclear0905@gmail.com

Cheng-Wei Lin

Department of Biochemistry and Molecular Biology, School of Medicine, College of

Medicine, Taipei Medical University,

$250 \mathrm{Wu}$ - Xing Street, Taipei, I I03I,

Taiwan, Republic of China

Email cwlin@tmu.edu.tw
Purpose: A lymph node (LN) yield $\geq 12$ is required to for accurate determination of nodal status for colorectal cancer but cannot always be achieved after neoadjuvant therapy. This study aims to determine the difference in LN yield from rectal cancer patients treated with and without neoadjuvant therapy and the effects of specific LN yields on survival.

Patients and Methods: The study cohort included a total of 4344 rectal cancer patients treated between January 2007 and December 2015, 2260 (52.03\%) of whom received neoadjuvant therapy. Data were retrieved from the Taiwan nationwide cancer registry database. The minimum acceptable LN yield below 12 was investigated using the maximum area under the ROC curve.

Results: The median LN yield was 12 (8-17) for patients who received neoadjuvant therapy and 17 (13-24) for those who did not. The recommended LN yield $\geq 12$ was achieved in $82.73 \%$ of patients without and $57.96 \%$ of those with neoadjuvant therapy $(p<0.0001)$. Patients with $\mathrm{LN}$ yield $\geq 12$ had a higher OS probability than did those with $\mathrm{LN}<12$ (OR, 1.33 ; $95 \%$ CI, $1.06-1.66 ; p=0.0124)$. However, the predictive accuracy for survival was greater for $\mathrm{LN}$ yield $\geq 10$ (AUC, 0.7767 ) than cut-offs of 12,8 , or 6 , especially in patients with pathologically-negative nodes (AUC, 0.7660).

Conclusion: Neoadjuvant therapy significantly reduces the LN yield in subsequent surgery. A lower yield $(\mathrm{LN} \geq 10)$ may be adequate for nodal evaluation in rectal cancer patients after neoadjuvant therapy.

Keywords: rectal cancer, neoadjuvant therapy, lymph node yield, quality, survival

\section{Introduction}

Metastatic nodal status is still the major prognostic factor in colorectal cancer management. ${ }^{1}$ Patients may experience stage migration and subsequent underestimation of disease severity if an adequate yield of lymph nodes (LN) is not obtained. ${ }^{2}$ Previous studies have confirmed that the LN yield correlates with patient outcomes, and a minimum of $12 \mathrm{LN}$ has been recommended as the standard yield by the American Joint Committee on Cancer (AJCC), the Union for International Cancer Control, and the College of American Pathologists (CAP). ${ }^{3}$

Neoadjuvant therapy followed by total mesorectal excision is currently the standard treatment for patients with stage II/III rectal cancer. ${ }^{4}$ However, neoadjuvant therapy for rectal cancer results in a lower LN yield, and yields below the suggested $12 \mathrm{LN}$ are reported in many studies. ${ }^{5-7}$ Mechera et al observed that rectal cancer patients receiving surgery with neoadjuvant therapy had a mean reduction of 3.9 LN and an average reduction in harvested positive LN of 0.7 compared to those receiving surgery without neoadjuvant therapy. ${ }^{2}$ Thus, the well-accepted quality 
metric for nodal evaluation has yielded inconclusive results for rectal cancer patients after neoadjuvant therapy.

Given the differences in LN yield between rectal cancer patients treated with and without preoperative chemoradiation, we hypothesized that the optimal LN yield cut-off point may differ between these groups of patients. Using data from the Taiwan nationwide cancer registry database, the primary aim of this study is to determine the differences in LN yield between rectal cancer patients treated with and without neoadjuvant therapy and the association between a LN yield of 12 and survival. A secondary aim is to identify the minimum acceptable LN yield using the maximum area under the Receiver operating characteristic (ROC) curve.

\section{Patients and Methods}

\section{Study Design and Participants}

The Taiwan Cancer Registry and National Health Insurance Research database were used to identify patients with a rectal cancer diagnosis and their associated cancer treatment such as surgery, chemotherapy, and radiation therapy. ${ }^{8}$ The Taiwan Cancer Registry database captures 97\% of the cancer cases in Taiwan and presents excellent data quality compared to other well-established cancer registries. ${ }^{9-11}$

Patients diagnosed with rectal cancer from January 2007 to December 2015 were identified using the following codes from the International Classification of Disease for Oncology third edition: rectosigmoid junction (code C19.9) and rectum (code $\mathrm{C} 20.9$ ); histologic type: adenocarcinoma (codes 8140, 8210, 8261, and 8263), mucinous adenocarcinoma (code 8480), or signet ring cell carcinoma (code 8490). These patients were all staged according to the classification system of the American Joint Committee on Cancer (AJCC), 7th edition. Follow-up began on the rectal cancer diagnosis date and ended on December 31, 2016. We included the most important confounding risk factors in this national cancer registry database: age, sex, histology type, tumor grade, clinical/pathological stage, margin status, LN yield, comorbid conditions, radiotherapy, year of cancer diagnosis, and adjuvant chemotherapy status. Comorbid conditions were graded for severity using the Charlson comorbidity index (CCI) score, as described previously. ${ }^{12,13}$ Patients without clear coding were excluded from our analysis. Patients with a history of cancer or metastatic disease were also excluded. Finally, data from a total of 4344 rectal cancer patients treated with or without neoadjuvant therapy were used for analysis in this retrospective, cross-sectional study (Figure 1). To measure the association between LN yield and survival of patients receiving neoadjuvant therapy, the overall survival (OS) probability was evaluated for each patient receiving follow-up for at least 1 year and up to 5 years.

\section{Statistical Analysis}

Categorical variables are presented as the frequency with percentage and compared using Pearson's chi-square test. Follow-up duration is shown as median and inter-quantile ranges and compared between two groups using the Wilcoxon rank sum test. Logistic regression analysis was used to estimate the associated OS probability for patients treated with neoadjuvant therapy between nodal yield $<12$ and $\geq 12$ after adjusting for all confounders. For patients receiving neoadjuvant therapy, the predicted model classification of $\mathrm{LN}$ yield less than $12(10,8,6,4$, or 2$)$ was also assessed using the maximum area under the ROC curve and compared with nodal yield $\geq 12$. AUCs were compared using DeLong's test. ${ }^{14}$ All analyses were conducted using SAS statistical software version 9.4 (SAS Institute, Inc., Cary, NC, USA). Statistical significance was set at $\mathrm{p}<0.05$.

\section{Results}

Relevant clinicopathological details are summarized in Table 1. From 2007 to 2015, a total of 4344 rectal cancer patients $(65.65 \%$ men) with a curable resection were included in the database. The mean age at diagnosis was $61 \pm 12$ years, and the median (Q1-Q3) follow-up time was 3.19 years. Of these patients, $2260(52.03 \%)$ received neoadjuvant therapy and $2084(47.97 \%)$ did not. The adjuvant chemotherapy administration rate was $28.27 \%$ among those who received preoperative neoadjuvant therapy and 50.24\% among those who did not. In the whole cohort, $30.16 \%$ of the patients had fewer than $12 \mathrm{LN}$ harvested. Patients who received neoadjuvant therapy were more likely to be male, younger than 65 , and have adenocarcinoma, well-differentiated tumor, advanced clinical stage, negative margin status, and s lower LN yield.

The median LN yield (Q1-3) was 12 (range, 8-17) in patients who received neoadjuvant therapy and 17 (range, 13-24) in those who did not (Figure 2A). Neoadjuvant therapy significantly decreased the LN yield, by $29.4 \%$ (5/ 17). Rectal cancer patients with neoadjuvant therapy tended to have fewer positive lymph nodes than did those without (Figure 2B). Twelve or more LN were harvested from $82.73 \%$ of the patients without 


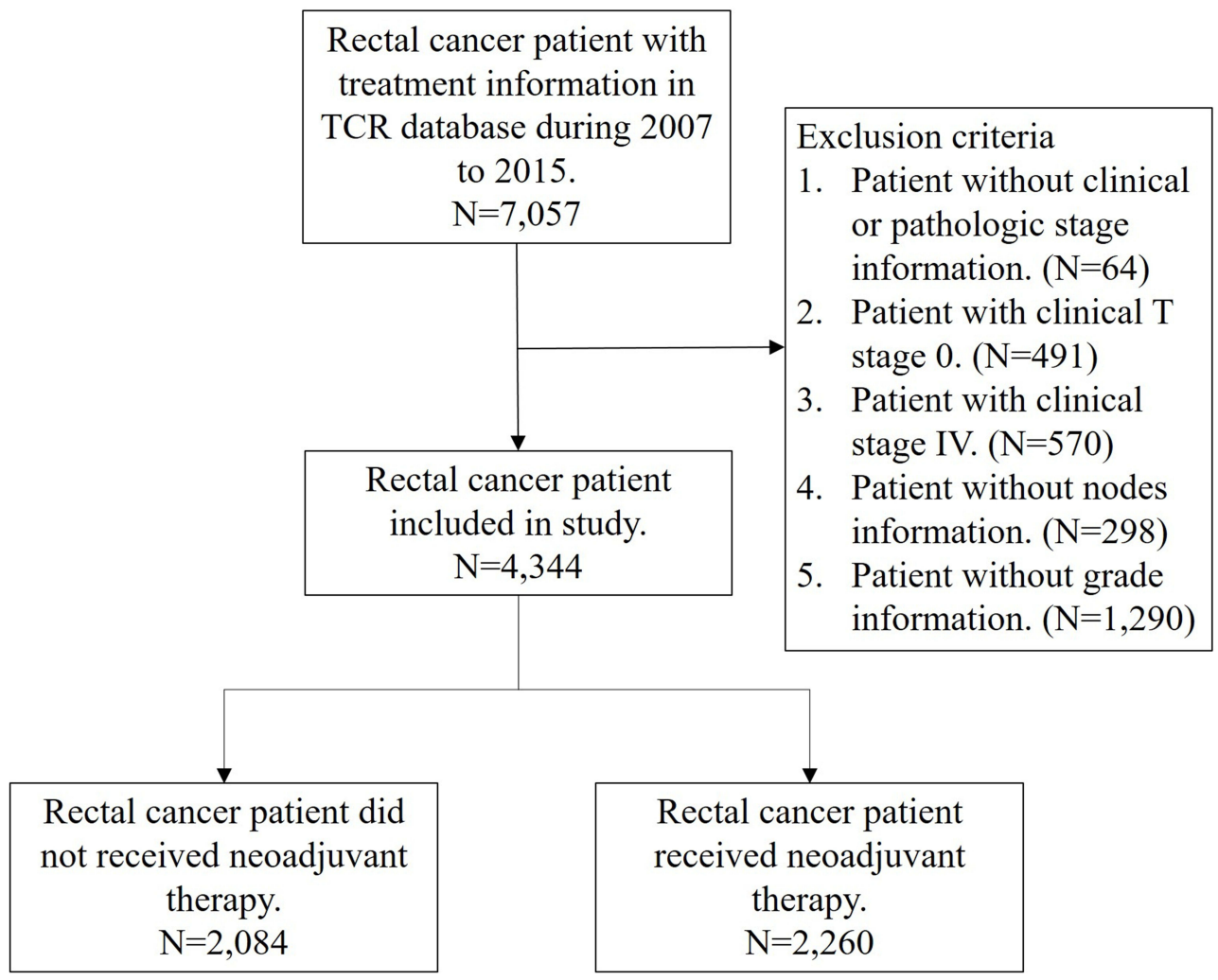

Figure I Study patient flow chart.

neoadjuvant therapy and $57.96 \%$ of those with neoadjuvant therapy $(p<0.0001)$ (Table 1). Clinicopathological characteristics of patients in the neoadjuvant group according to $\mathrm{LN}$ yield $(<12$ and $\geq 12)$ are shown in Supplementary Table 1 . The mean survival time during follow up between rectal cancer patients receiving neoadjuvant therapy or not was 3.82 and 3.99 years. Comparison of adjusted odds ratio for 5-year survival probability for patients between those who did and did not receive neoadjuvant therapy, according to LN yield, are shown in Supplementary Table 2.

In patients who received neoadjuvant therapy, univariable and multivariable analysis revealed that significant risk factors for poor OS probability included $\mathrm{LN}<12$, age $>65$ years, poorly differentiated or undifferentiated tumor grade, positive margin, advanced stage, and severe comorbid conditions and without adjuvant chemotherapy (Table 2). Because neoadjuvant therapy was associated with a lower $\mathrm{LN}$ yield, we investigated the optimal number of $\mathrm{LN}$ below 12 . Using a range of $\mathrm{LN}$ yield cut-off points $(12,10,8,6,4$, and 2), significant differences in the OS probability were found between $\mathrm{LN}$ yield $\geq 12,10,8$, and 6. However, LN yield $\geq 10$ provided better predictive accuracy for survival with a higher AUC (0.7767) than did the other tested cut-offs, especially in node-negative patients (AUC:0.7660) (Table 3).

\section{Discussion}

In this large-cohort study, we observed that the LN yield was significantly lower in rectal cancer patients who received neoadjuvant therapy than in those who did not. The OS probabilities differed significantly between patients with $L N$ yield $\geq 12$ and $<12$, as revealed by univariate and multivariable analysis. For LN yield cut-off points below 12 , $\mathrm{LN}$ yield $\geq 10$ provided the best predictive accuracy for survival, especially in patients with pathologically negative nodes. Thus, a lower value (LN yield $\geq$ 10) may an acceptable cut-off point for rectal cancer patients after neoadjuvant therapy.

This study has several strengths. First, the target group $(n=4344)$ was large, and the follow-up duration was sufficient for our results to be convincing. Second, our database provides important information on the predisposing factors that may influence survival (eg, grade, histology type, clinicopathological stage, margin status, comorbidities and adjuvant chemotherapy). An in-depth 
Table I Patient Clinicopathologic Characteristics

\begin{tabular}{|c|c|c|c|c|}
\hline Variables & N (\%) & $\begin{array}{c}\text { Did Not Received Neoadjuvant } \\
\text { Therapy }\end{array}$ & $\begin{array}{c}\text { Received Neoadjuvant } \\
\text { Group }\end{array}$ & $p$-value* \\
\hline Overall & $4344(100.00)$ & 2084 (47.97) & $2260(52.03)$ & \\
\hline \multicolumn{5}{|l|}{ Gender } \\
\hline Male & $2852(65.65)$ & $1315(63.10)$ & I537 (68.0I) & 0.0007 \\
\hline Female & 1492 (34.35) & $769(36.90)$ & $723(31.99)$ & \\
\hline \multicolumn{5}{|l|}{ Age groups } \\
\hline$\leqq 65$ & $2750(63.3 I)$ & $1258(60.36)$ & $1492(66.02)$ & 0.0001 \\
\hline$>65$ & $1594(36.69)$ & $826(39.64)$ & 768 (33.98) & \\
\hline \multicolumn{5}{|l|}{ Histology type } \\
\hline Adenocarcinoma & $4135(95.19)$ & I96I (94.10) & $2174(96.19)$ & 0.0054 \\
\hline Mucinous & $179(4.12)$ & $105(5.04)$ & 74 (3.27) & \\
\hline Signet & $30(0.69)$ & $18(0.86)$ & $12(0.53)$ & \\
\hline \multicolumn{5}{|l|}{ Grade } \\
\hline Well/Moderately & $3983(91.69)$ & $1878(90.12)$ & $2105(93.14)$ & 0.0003 \\
\hline Poorly/Undifferentiated & $36 I(8.3 I)$ & $206(9.88)$ & I55 (6.86) & \\
\hline \multicolumn{5}{|l|}{ Clinical T stage } \\
\hline $\mathrm{TI}$ & $67(1.54)$ & $56(2.69)$ & II (0.49) & $<0.0001$ \\
\hline $\mathrm{T} 2$ & $811(18.67)$ & $526(25.24)$ & $285(\mid 2.6 I)$ & \\
\hline T3 & $3004(69.15)$ & $1279(61.37)$ & I725 (76.33) & \\
\hline $\mathrm{T} 4$ & $462(10.64)$ & $223(10.70)$ & $239(10.58)$ & \\
\hline \multicolumn{5}{|l|}{ Clinical $\mathrm{N}$ stage } \\
\hline No & $1636(37.66)$ & $945(45.35)$ & $691(30.58)$ & $<0.0001$ \\
\hline $\mathrm{NI}$ & $1614(37.15)$ & 677 (32.49) & $937(41.46)$ & \\
\hline N2 & $1094(25.18)$ & $462(22.17)$ & $632(27.96)$ & \\
\hline \multicolumn{5}{|l|}{ Pathologic T stage } \\
\hline урТ0 & $293(6.74)$ & - & $293(12.96)$ & $<0.0001$ \\
\hline $\mathrm{pTI} /$ ypTI & $153(3.52)$ & $4 \mathrm{I}(1.97)$ & II2 (4.96) & \\
\hline $\mathrm{pT} 2 /$ урT2 & 755 (17.38) & $215(10.32)$ & $540(23.89)$ & \\
\hline рТ3/ урТ3 & $2702(62.20)$ & $1518(72.84)$ & II 84 (52.39) & \\
\hline $\mathrm{pT} 4 /$ yрT4 & $44 I(10.15)$ & $310(14.88)$ & $|3|(5.80)$ & \\
\hline \multicolumn{5}{|l|}{ Pathologic $N$ stage } \\
\hline pNo/ ypNo & $2020(46.50)$ & $546(26.20)$ & $1474(65.22)$ & $<0.0001$ \\
\hline $\mathrm{pNI}$ ypNI & $1373(31.61)$ & $839(40.26)$ & $534(23.63)$ & \\
\hline pN2 /ypN2 & $951(21.89)$ & $699(33.54)$ & $252(11.15)$ & \\
\hline \multicolumn{5}{|l|}{ Margin } \\
\hline Positive & $214(4.93)$ & $127(6.09)$ & $87(3.85)$ & 0.0006 \\
\hline Negative & $4 \mid 30(95.07)$ & $1957(93.91)$ & $2173(96.15)$ & \\
\hline \multicolumn{5}{|l|}{ Lymph node yield } \\
\hline$<12$ & $1310(30.16)$ & $360(17.27)$ & $950(42.04)$ & $<0.0001$ \\
\hline$\geq 12$ & $3034(69.84)$ & I 724 (82.73) & $1310(57.96)$ & \\
\hline \multicolumn{5}{|l|}{ Adjuvant chemotherapy } \\
\hline No & $2658(61.19)$ & $1037(49.76)$ & I62| (7I.73) & \\
\hline Yes & $1686(38.81)$ & 1047 (50.24) & $639(28.27)$ & $<0.0001$ \\
\hline
\end{tabular}

(Continued) 
Table I (Continued).

\begin{tabular}{|c|c|c|c|c|}
\hline Variables & N (\%) & $\begin{array}{c}\text { Did Not Received Neoadjuvant } \\
\text { Therapy }\end{array}$ & $\begin{array}{c}\text { Received Neoadjuvant } \\
\text { Group }\end{array}$ & $p$-value* \\
\hline \multicolumn{5}{|c|}{ Charlson comorbidity } \\
\hline $0-1$ & $3820(87.94)$ & $1813(87.00)$ & $2007(88.8 \mathrm{I})$ & 0.0492 \\
\hline $2-3$ & $435(10.01)$ & $232(11.13)$ & $203(8.98)$ & \\
\hline$\geqq 4$ & $89(2.05)$ & $39(1.87)$ & $50(2.21)$ & \\
\hline \multicolumn{5}{|l|}{ Surgery type } \\
\hline APR & $746(17.17)$ & $334(16.03)$ & $412(18.23)$ & $<0.0001$ \\
\hline LAR & $3007(69.22)$ & I492 (7I.59) & $1515(67.04)$ & \\
\hline Protectomy & 499 (II.49) & $|8|$ (8.69) & $318(14.07)$ & \\
\hline Unknown & $92(2.12)$ & 77 (3.69) & $15(0.66)$ & \\
\hline \multicolumn{5}{|c|}{ Follow-up period, year } \\
\hline Median (QI-Q3) & $3.19(1.62-5.29)$ & $3.27(1.75-5.20)$ & $3.11(1.49-5.36)$ & 0.2103 \\
\hline \multicolumn{5}{|c|}{$\begin{array}{l}\text { Survival time during follow-up } \\
\text { period, year }\end{array}$} \\
\hline Mean (SD) & $3.90(1.34)$ & $3.99(1.28)$ & $3.82(1.39)$ & 0.0002 \\
\hline
\end{tabular}

Note: *P-value was calculated from Pearson's chi-square.

assessment of the effects of these factors on survival was performed. Third, in contrast to previous studies, we aimed to determine the association between survival probability and a range of LN yield cut-off points below 12; thus this study is more relevant to actual clinical practice.

Regional lymph node metastasis is considered one of the most important prognostic indicators for outcomes in all cancer patients, including colorectal cancer. ${ }^{15}$ For decades, the N-staging system based on the numbered lymph nodes was used to guide decisions concerning adjuvant treatment. ${ }^{16}$ As such, it was first recommended at the World Congress of Gastroenterology in 1990 that a minimum number of 12 lymph nodes should be examined as an important quality metric for adequate LN dissection for optimal staging of patients. Recent studies report that LN count or LN yield may serve as a prognostic factor in colorectal cancer patients. ${ }^{15,17,18} \mathrm{In}$ a systematic review that included 61,371 colon cancer patients, Chang et al reported that the number of lymph nodes evaluated after surgical resection was positively associated with survival of patients with stage II-III colon cancer. ${ }^{5}$ Ecker et al also demonstrated that greater LN assessment was associated with an increased likelihood of nodal involvement. ${ }^{18}$ In node-negative patients, greater LN assessment was associated with a decreased risk of death, with the largest actuarial survival differences observed for $\geq 15 \mathrm{LN}$. Therefore, the extent of LN identification has prognostic significance in colorectal cancer surgery and supports consideration of the number of lymph nodes evaluated as a measure of the quality of colorectal cancer care.

In recent decades, neoadjuvant therapy followed by curative surgery has become the standard management protocol for rectal cancer. Previous studies report that preoperative therapy may decrease the number of lymph nodes harvested. Similarly, Amajoyi et al reported that the mean number of lymph nodes retrieved was lower in patients treated with neoadjuvant therapy than in those without. ${ }^{19}$ In another study, rectal cancer patients treated with neoadjuvant therapy had significantly fewer LN assessed, and the current recommendation of at least 12 LN was met in only $37 \%$ of these patients. ${ }^{20}$ Rullier et al also reported that rectal cancer patients after chemoradiation had a lower mean number of lymph nodes retrieved (17 vs $13 \mathrm{LN}$ ) and a lower mean number of positive lymph nodes $(2.3$ vs $1.2 \mathrm{LN}){ }^{21}$ Consistent with this evidence, the results of the present study indicated that the median LN yield (Q1-3) was 12 (range, 8-17) for patients who received neoadjuvant therapy and 17 (range, 13-24) for patients who did not. After preoperative therapy, the LN yield reduced significantly by about $29.4 \%$ and only $1310(57.96 \%)$ of patients had $\geq 12 \mathrm{LN}$ assessed. Therefore, in addition to its direct effect on the primary tumor, the neoadjuvant therapy may also reduce the number of lymph nodes retrieved from rectal cancer specimens. 

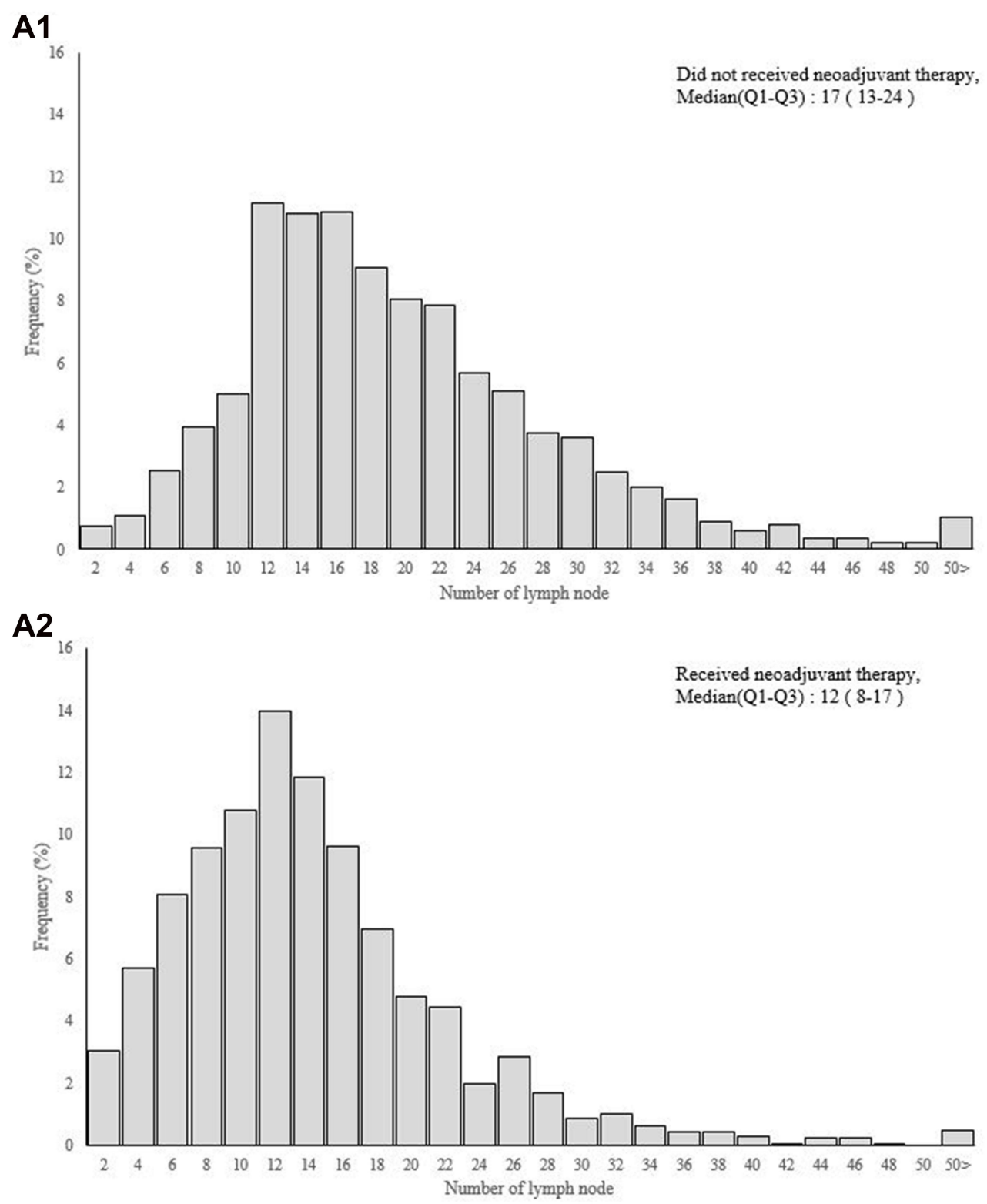

Figure 2 Continue.

Although the LN yield achieved for rectal cancer is associated with survival, its impact as a prognostic factor is still debated, especially for patients who received neoadjuvant therapy. A possible explanation is that a decreased LN yield may be reflective of responsiveness to preoperative therapy and thereby is associated with greater survival. Rullier et al reported that the number of lymph nodes retrieved was not associated with disease-free survival or overall survival among 198 patients with pathological lymph node-negative disease who received neoadjuvant therapy. ${ }^{21}$ Among patients who received neoadjuvant therapy, Kim et al observed that the number of lymph nodes retrieved was not significantly associated with recurrence or survival of lymph-node-negative rectal cancer. ${ }^{22}$ In a cohort of 1680 stage II/II rectal cancer patients with data in the National Comprehensive Cancer Network prospective oncology database, Abdel-Misih et al also reported that $\mathrm{LN}$ yield and status were not significant prognosticators of overall survival in multivariable analysis. ${ }^{3}$ Their findings further support that LN yield in rectal cancer is multifactorial and the nodal metric may not be clinically relevant after neoadjuvant management. However, a Danish population-based study of 6793 patients with rectal cancer found that $\mathrm{LN}$ yield is an 


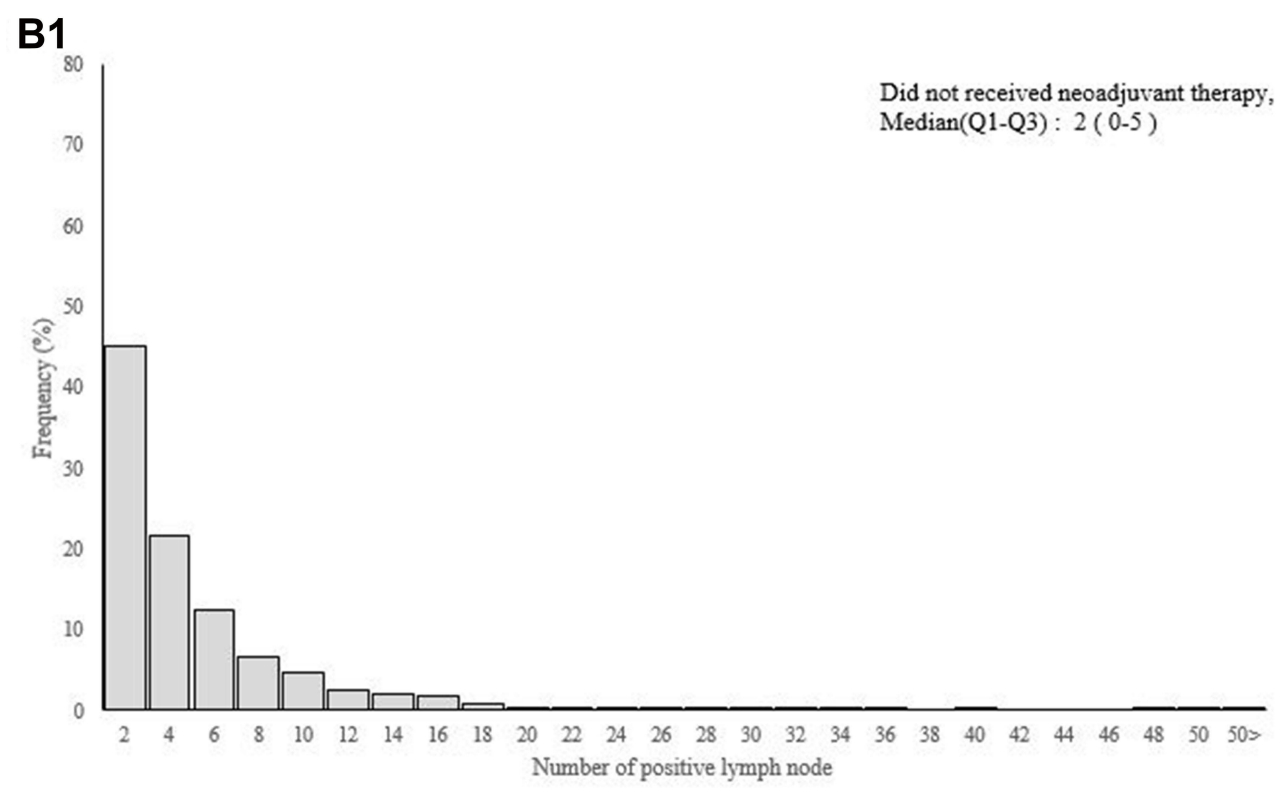

B2

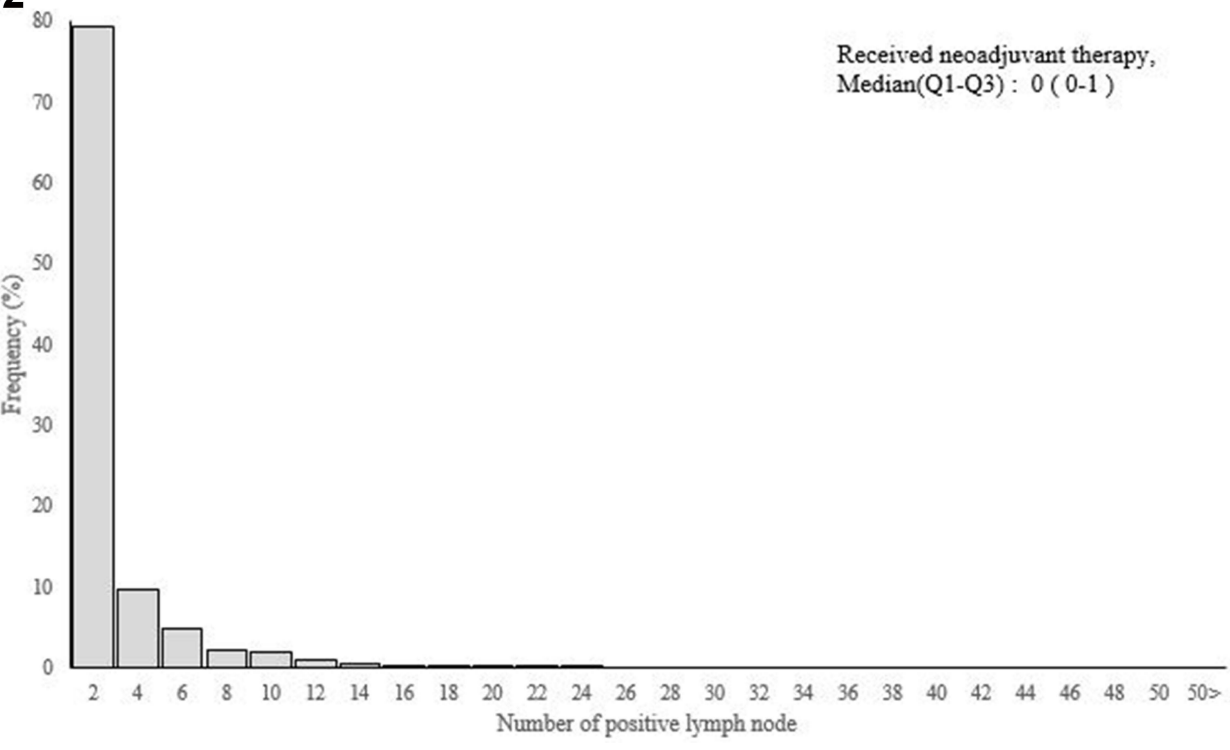

Figure 2 (A) Number of lymph node and (B) positive lymph node between rectal cancer patients receiving neoadjuvant therapy or not.

independent prognostic factor for rectal cancer, regardless of neoadjuvant therapy status. ${ }^{23}$ Regression analysis in the present study revealed significant differences in OS probability between the neoadjuvant patients with $<12 \mathrm{LN}$ vs $\geq$ $12 \mathrm{LN}$. Using lower-value cut-off points, the OS probabilities between $\mathrm{LN}$ yield $\geq 12,10,8,6$ still differ significantly. However, LN yield $\geq 10$ provided better predictive accuracy for survival, with a higher AUC (0.7767), than did the other tested cut-offs (LN yield $\geq 12$, 8, or 6 ), especially in patients with pathologically-negative nodes (AUC, 0.7660). Although previous studies report no significant association between 5 -year survival and lymph node count, node positivity is proportional to number of nodes. ${ }^{20,24}$ These results indicate that retrieving an adequate number of LN in patients with neoadjuvant therapy is still important for accurate staging. Inadequate LN evaluation may lead to underestimation of positive nodal status and stage migration, which may affect the decision to use adjuvant chemotherapy. Thus, considering the importance of LN yield on nodal evaluation, a lower LN yield is more reflective of patient status in those with neoadjuvant therapy than in those without, and LN yield $\geq 10$ may be clinically relevant as a nodal metric for determining the standard of care. 
Table 2 Univariable and Multivariable Logistic Regression Analyses of Clinicopathologic Features to Overall Survival in Rectal Cancer Patients Receiving Neoadjuvant Therapy (N=2260)

\begin{tabular}{|c|c|c|c|c|}
\hline \multicolumn{2}{|l|}{ Variables } & \multirow{4}{*}{$\begin{array}{c}\text { Univariate } 95 \% \mathrm{Cl} \\
\text { Ref } \\
1.20(0.99-1.46)\end{array}$} & \multirow{4}{*}{$\begin{array}{c}\text { Multivariable }^{\mathrm{a}} 95 \% \mathrm{Cl} \\
\text { Ref } \\
1.33(1.06-1.66)\end{array}$} & \multirow{4}{*}{$\begin{array}{l}P \text {-value } \\
0.0124\end{array}$} \\
\hline Lymph node yield & & & & \\
\hline & $<12$ & & & \\
\hline & $\geq 12$ & & & \\
\hline \multirow[t]{3}{*}{ Gender } & & & & \\
\hline & Male & $0.86(0.69-1.06)$ & $0.79(0.63-1.01)$ & 0.0548 \\
\hline & Female & Ref. & Ref. & \\
\hline \multirow[t]{3}{*}{ Age groups } & & & & \\
\hline & $\leq 65$ & Ref. & Ref. & \\
\hline & $>65$ & $0.64(0.53-0.78)$ & $0.61(0.49-0.76)$ & $<0.0001$ \\
\hline \multirow[t]{3}{*}{ Grade } & & & & \\
\hline & Well/Moderately & Ref. & Ref. & \\
\hline & Poorly/ Undifferentiated & $0.27(0.20-0.38)$ & $0.40(0.27-0.58)$ & $<0.0001$ \\
\hline \multirow[t]{6}{*}{ Pathologic T stage } & & & & \\
\hline & урт0 & Ref. & Ref. & \\
\hline & урTI & $0.95(0.38-1.84)$ & $0.90(0.4 I-1.94)$ & 0.7777 \\
\hline & урТ2 & $0.60(0.31-1.17)$ & $0.59(0.36-0.97)$ & 0.0373 \\
\hline & урТ3 & $0.25(0.13-0.47)$ & $0.28(0.18-0.45)$ & $<0.0001$ \\
\hline & yрT4 & $0.11(0.05-0.23)$ & $0.17(0.09-0.31)$ & $<0.0001$ \\
\hline \multirow[t]{4}{*}{ Pathologic N stage } & & & & \\
\hline & ypNo & Ref. & Ref. & \\
\hline & ypNI & $0.51(0.40-0.64)$ & $0.63(0.49-0.81)$ & 0.0004 \\
\hline & ypN2 & $0.23(0 . \mid 8-0.31)$ & $0.32(0.23-0.45)$ & $<0.0001$ \\
\hline \multirow[t]{4}{*}{ Charlson comorbidity } & & & & \\
\hline & $0-1$ & Ref. & Ref. & \\
\hline & $2-3$ & $0.70(0.5 \mathrm{I}-0.97)$ & $0.65(0.46-0.93)$ & 0.0195 \\
\hline & $\geqq 4$ & $0.64(0.35-1.17)$ & $0.49(0.25-0.98)$ & 0.0421 \\
\hline \multirow[t]{3}{*}{ Margin } & & & & \\
\hline & Positive & $0.26(0.17-0.4 I)$ & $0.36(0.22-0.60)$ & $<0.0001$ \\
\hline & Negative & Ref. & Ref. & \\
\hline \multirow[t]{2}{*}{ Adjuvant chemotherapy } & No & $0.85(0.69-1.05)$ & $0.68(0.54-0.87)$ & 0.0020 \\
\hline & Yes & Ref. & Ref. & \\
\hline \multirow[t]{5}{*}{ Surgery type } & & & & \\
\hline & APR & $0.6 \mathrm{I}(0.48-0.77)$ & $0.79(0.60-1.04)$ & 0.096 \\
\hline & LAR & Ref. & Ref. & \\
\hline & Protectomy & $0.78(0.25-2.46)$ & $0.76(0.21-2.74)$ & 0.6757 \\
\hline & Unknown & $0.93(0.70-1.24)$ & $\mathrm{I} .02(0.75-\mid .4 \mathrm{I})$ & 0.8857 \\
\hline
\end{tabular}

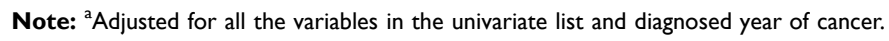

Notably, the prognostic value of nodal parameters other than LN yield is under investigation, including the ratio of positive lymph nodes ( $\mathrm{rN}$ ) and the log odds of positive lymph nodes (LODDS). As discussed in our previous report, the LODDS provides a more accurate survival prediction than does $\mathrm{LN}$ yield or $\mathrm{rN}$ in oral cancer patients. ${ }^{25,26}$ LODDS can discriminate between patients who have the same ratio of node metastasis, especially in patients without positive lymph nodes or with an insufficient number of retrieved nodes. However, the standard cutoff points for both $\mathrm{rN}$ and LODDS are not well defined, making these nodal systems difficult to use in clinical practice. Moreover, neoadjuvant therapy significantly reduces the LN yield and number of positive lymph 


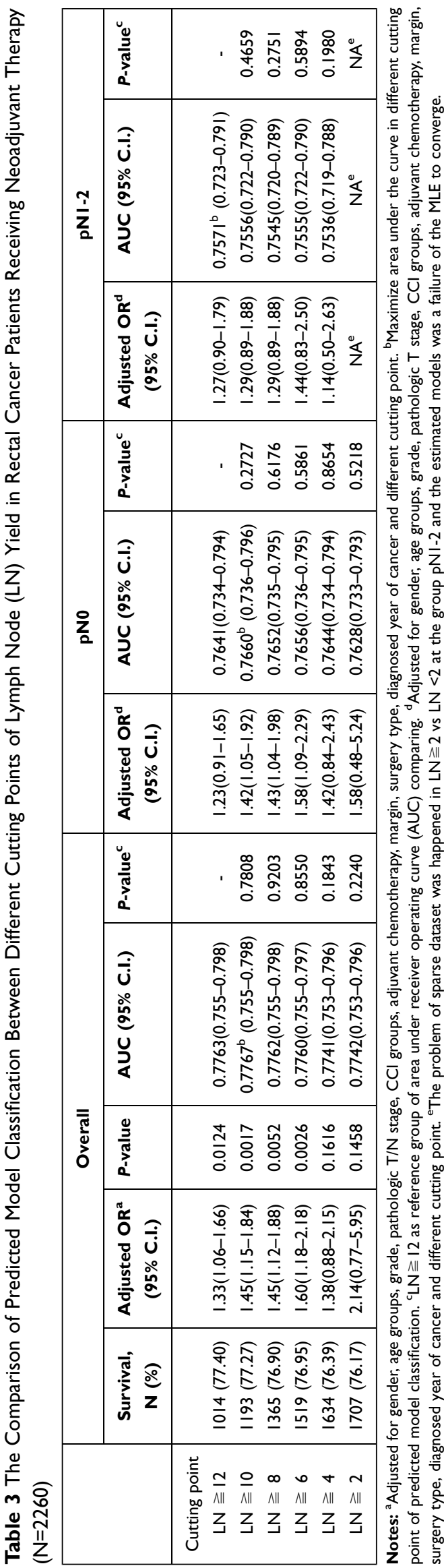

nodes in rectal cancer patients (Figure 2A and B). Using the $\mathrm{rN}$ or LODDS system alone would not ascertain adequate $\mathrm{LN}$ retrieval for optimal cancer staging. In our neoadjuvant therapy group, we observed that the change in $\mathrm{rN}$ is limited when the $\mathrm{LN}$ yield is inadequate $(\mathrm{LN} \leq 8)$ (Supplementary Figure 1). This study did not aim to challenge the prognostic value of $\mathrm{rN}$ or LODDS but rather to determine whether a lower LN would be an acceptable quality metric of nodal evaluation and could also be used for survival prediction.

This study has several limitations. First, different coding quality between hospitals may result in bias. However, the National Health Insurance institution systematically reviews charts to verify the accuracy of diagnosis and treatment coding. Second, the TCR database does not include some important clinicopathological characteristics, such as perineural invasion, quality of TME specimen, neoadjuvant therapy regimens and dose, patient compliance with neoadjuvant therapy or adjuvant chemotherapy, pathological assessment, and actual tumor regression. This lack of information could potentially cause bias. However, a previous study reported no significant differences in the number of lymph nodes harvested between different pathologists. ${ }^{27}$ Lastly, information regarding the precise treatment protocols for neoadjuvant therapy and adjuvant chemotherapy was not available. A retrospective study and a prospective clinical study with the same heterogeneity of rectal cancer patients are necessary to confirm our findings.

\section{Conclusion}

Neoadjuvant therapy significantly reduces the number of LN retrieved and positive LN during surgery for rectal cancer. Although the current recommended LN yield $(\geq 12)$ is a prognostic factor for OS, a lower number of nodes $(\geq 10)$ provides more accurate survival prediction than other tested cut-offs, especially in patients with pathologically-negative lymph nodes.

\section{Abbreviations}

LN, lymph node; AJCC, American Joint Committee on Cancer; CAP, Union for International Cancer Control, and College of American Pathologists; TME, total mesorectal excision; ROC, receiver operating characteristic; TCR, Taiwan Cancer Registry; NHIRD, National Health Insurance Research database; ICD-O-3, International Classification of Diseases for Oncology, 3rd Edition; CCI, 
Charlson comorbidity index; OS, overall survival; rN, positive lymph nodes; LODDS, log odds of positive lymph nodes.

\section{Ethics Approval and Informed Consent}

This study was conducted according to the guidelines of the Declaration of Helsinki and approved by the Ethics Committee of the Institutional Review Board of the Chi Mei Medical Center (IRB: CMFHR10707-012). This study had a non-interventional retrospective design; no human subjects or personally identifying information were used, and all data were analyzed anonymously. Thus, informed consent was waived by the IRB.

\section{Acknowledgments}

We acknowledge the support provided by the following grants: (1) Health and Welfare surcharge on tobacco products (MOHW110-TDU-B-212-144020, WanFang Hospital, Chi-Mei Medical Center, and Hualien Tzu-Chi Hospital Joing Cancer Center Grant-Focus on Colon Cancer Research); (2) CMFHR11011 and 110CM-TMU -01 from the Chi Mei Medical center. We are also grateful to the Health Data Science Center, National Cheng Kung University Hospital for providing administrative and technical support.

\section{Funding}

The author(s) received no financial support for the research, authorship, and/or publication of this article.

\section{Disclosure}

The authors have no conflicts of interest.

\section{References}

1. Thorn CC, Woodcock NP, Scott N, Verbeke C, Scott SB, Ambrose NS. What factors affect lymph node yield in surgery for rectal cancer? Colorectal Dis. 2004;6(5):356-361. doi:10.1111/j.1463-1318.2004.00 670.x

2. Mechera R, Schuster T, Rosenberg R, Speich B. Lymph node yield after rectal resection in patients treated with neoadjuvant radiation for rectal cancer: a systematic review and meta-analysis. Eur J Cancer. 2017;72:84-94. doi:10.1016/j.ejca.2016.10.031

3. Abdel-Misih SR, Wei L, Benson AB 3rd, et al. Neoadjuvant therapy for rectal cancer affects lymph node yield and status without clear implications on outcome: the case for eliminating a metric and using preoperative staging to guide therapy. J Natl Compr Canc Netw. 2016;14(12):1528-1534. doi:10.6004/jncen.2016.0164

4. Bosset JF, Collette L, Calais G, et al. Chemotherapy with preoperative radiotherapy in rectal cancer. $N$ Engl J Med. 2006;355(11):1114-1123. doi:10.1056/NEJMoa060829
5. Chan DKH, Tan KK. Lower lymph node yield following neoadjuvant therapy for rectal cancer has no clinical significance. $J$ Gastrointest Oncol. 2019;10(1):42-47. doi:10.21037/jgo.2018.10.02

6. Doll D, Gertler R, Maak M, et al. Reduced lymph node yield in rectal carcinoma specimen after neoadjuvant radiochemotherapy has no prognostic relevance. World J Surg. 2009;33(2):340-347. doi:10. 1007/s00268-008-9838-8

7. Morcos B, Baker B, Al Masri M, Haddad H, Hashem S. Lymph node yield in rectal cancer surgery: effect of preoperative chemoradiotherapy. Eur J Surg Oncol. 2010;36(4):345-349. doi:10.1016/j.ejso.2009.12.006

8. Liang YH, Shao YY, Chen HM, Cheng AL, Lai MS, Yeh KH. Irinotecan and oxaliplatin might provide equal benefit as adjuvant chemotherapy for patients with resectable synchronous colon cancer and liver-confined metastases: a nationwide database study. Anticancer Res. 2017;37(12):7095-7104.

9. Bray F, Parkin DM. Evaluation of data quality in the cancer registry: principles and methods. Part I: comparability, validity and timeliness. Eur J Cancer. 2009;45(5):747-755. doi:10.1016/j. ejca.2008.11.032

10. Chiang CJ, Lo WC, Yang YW, You SL, Chen CJ, Lai MS. Incidence and survival of adult cancer patients in Taiwan, 2002-2012. J Formos Med Assoc. 2016;115(12):1076-1088. doi:10.1016/j.jfma.2015.10. 011

11. Chiang NJ, Chen LT, Tsai CR, Chang JS. The epidemiology of gastrointestinal stromal tumors in Taiwan, 1998-2008: a nationwide cancer registry-based study. BMC Cancer. 2014;14:102. doi:10.1186/1471-2407-14-102

12. Huang Y, Zhang Y, Li J, Liu G. Charlson comorbidity index for evaluation of the outcomes of elderly patients undergoing laparoscopic surgery for colon cancer. J BUON. 2017;22(3):686-691.

13. Tian Y, Xu B, Yu G, Li Y, Liu H. Age-adjusted charlson comorbidity index score as predictor of prolonged postoperative ileus in patients with colorectal cancer who underwent surgical resection. Oncotarget. 2017;8(13):20794-20801. doi:10.18632/oncotarget.15285

14. DeLong ER, DeLong DM, Clarke-Pearson DL. Comparing the areas under two or more correlated receiver operating characteristic curves: a nonparametric approach. Biometrics. 1988;44(3):837-845. doi:10. $2307 / 2531595$

15. Chang GJ, Rodriguez-Bigas MA, Skibber JM, Moyer VA. Lymph node evaluation and survival after curative resection of colon cancer: systematic review. J Natl Cancer Inst. 2007;99(6):433-441. doi:10. 1093/jnci/djk092

16. Benson AB, Venook AP, Al-Hawary MM, et al. NCCN guidelines insights: rectal cancer, version 6.2020. J Natl Compr Canc Netw. 2020;18(7):806-815. doi:10.6004/jnccn.2020.0032

17. Cianchi F, Palomba A, Boddi V, et al. Lymph node recovery from colorectal tumor specimens: recommendation for a minimum number of lymph nodes to be examined. World J Surg. 2002;26(3):384-389. doi:10.1007/s00268-001-0236-8

18. Ecker BL, McMillan MT, Datta J, et al. Lymph node evaluation and survival after curative-intent resection of duodenal adenocarcinoma: a matched cohort study. Eur J Cancer. 2016;69:135-141. doi:10.10 16/j.ejca.2016.09.027

19. Amajoyi R, Lee Y, Recio PJ, Kondylis PD. Neoadjuvant therapy for rectal cancer decreases the number of lymph nodes harvested in operative specimens. Am J Surg. 2013;205(3):289-292; discussion 292. doi:10.1016/j.amjsurg.2012.10.020

20. Govindarajan A, Gonen M, Weiser MR, et al. Challenging the feasibility and clinical significance of current guidelines on lymph node examination in rectal cancer in the era of neoadjuvant therapy. J Clin Oncol. 2011;29(34):4568-4573. doi:10.1200/JCO.2011.37.2235

21. Rullier A, Laurent C, Capdepont M, et al. Lymph nodes after preoperative chemoradiotherapy for rectal carcinoma: number, status, and impact on survival. Am J Surg Pathol. 2008;32(1):45-50. doi:10.1097/PAS.0b013e3180dc92ab 
22. Kim YW, Kim NK, Min BS, et al. The prognostic impact of the number of lymph nodes retrieved after neoadjuvant chemoradiotherapy with mesorectal excision for rectal cancer. J Surg Oncol. 2009;100(1):1-7. doi:10.1002/jso.21299

23. Lykke J, Jess P, Roikjaer O; Danish Colorectal Cancer G. Increased lymph node yield is associated with improved survival in rectal cancer irrespective of neoadjuvant treatment: results from a national cohort study. Dis Colon Rectum. 2015;58(9):823-830. doi:10.1097/ DCR.0000000000000429

24. Persiani R, Biondi A, Gambacorta MA, et al. Prognostic implications of the lymph node count after neoadjuvant treatment for rectal cancer Br J Surg. 2014;101(2):133-142. doi:10.1002/bjs.9341
25. Lee CC, Su YC, Hung SK, et al. Recommendation for incorporation of a different lymph node scoring system in future AJCC N category for oral cancer. Sci Rep. 2017;7(1):14117. doi:10.1038/s41598-017-06452-0

26. Lee CC, Lin YS, Kang BH, et al. Incorporation of $\log$ odds of positive lymph nodes into the AJCC TNM classification improves prediction of survival in oral cancer. Clin Otolaryngol. 2017;42 (2):425-432. doi:10.1111/coa.12809

27. Marks JH, Valsdottir EB, Rather AA, Nweze IC, Newman DA, Chernick MR. Fewer than 12 lymph nodes can be expected in a surgical specimen after high-dose chemoradiation therapy for rectal cancer. Dis Colon Rectum. 2010;53(7):1023-1029. doi:10.1007/ DCR.0b013e3181dadeb4

\section{Publish your work in this journal}

Cancer Management and Research is an international, peer-reviewed open access journal focusing on cancer research and the optimal use of preventative and integrated treatment interventions to achieve improved outcomes, enhanced survival and quality of life for the cancer patient
The manuscript management system is completely online and includes a very quick and fair peer-review system, which is all easy to use. Visit http://www.dovepress.com/testimonials.php to read real quotes from published authors. 